\title{
Semiclassical analysis of spectral singularities and their applications in optics
}

\author{
Ali Mostafazadeh* \\ Department of Mathematics, Koç University, Sariyer, Istanbul 34450, Turkey
}

(Received 29 March 2011; published 8 August 2011)

\begin{abstract}
Motivated by possible applications of spectral singularities in optics, we develop a semiclassical method of computing spectral singularities. We use this method to examine the spectral singularities of a planar slab gain medium whose gain coefficient varies due to the exponential decay of the intensity of the pumping beam inside the medium. For both singly and doublypumped samples, we obtain universal upper bounds on the decay constant beyond which no lasing occurs. Furthermore, we show that the dependence of the wavelength of the spectral singularities on the value of the decay constant is extremely mild. This is an indication of the stability of optical spectral singularities.
\end{abstract}

DOI: 10.1103/PhysRevA.84.023809

PACS number(s): 42.25.Bs, 03.65.Nk, 24.30.Gd, 42.60.Da

\section{INTRODUCTION}

We say that an $n \times n$ matrix $\mathbf{A}$ is diagonliazable if we can expand every $n$-dimensional column vector as a linear combinations of the eigenvectors of $\mathbf{A}$. In this case we can construct a basis consisting of these eigenvectors and diagonalize $A$ by transforming to this basis. We can use this terminology for linear operators acting in infinite-dimensional Hilbert spaces [1]. In quantum mechanics we make heavy use of the fact that Hermitian operators are diagonalizable. Naturally, there are non-Hermitian operators that do not have this property. For non-Hermitian Schrödinger operators whose spectrum has a continuous part, a source of non-diagonliazability of the operator is the presence of what mathematicians call a spectral singularity [2]. Spectral singularities have been extensively studied by mathematicians since the 1950s [3] but remained essentially unknown to physicists until recently [4].

During the past 10 years or so it was noticed that some nonHermitian operators can be used to serve as the Hamiltonian for a unitary quantum system provided that one modifies the inner product of the Hilbert space [5]. Spectral singularities emerge as an obstruction for the implementation of this Hermitization procedure for non-Hermitian scattering Hamiltonians. This was initially noticed in the study of complex point interactions [6,7]. In Ref. [4], we give the physical meaning of spectral singularities by identifying them with the energies of certain scattering states that behave exactly like resonances; they correspond to zero-width resonances [8,9]. In [4] and [8] we propose optical realizations of spectral singularities, and in [10] we use a simple toy model to show that the optical spectral singularities give rise to a lasing effect that takes place exactly at the threshold gain. The calculations of spectral singularities given in [4], [8], and [10] rely on the assumption that the gain coefficient is constant throughout the gain region. This allows for an essentially exact and analytic treatment of the problem, but it is practically unattainable. In the present article, we develop a systematic semiclassical treatment of spectral singularities and examine its application for more realistic optical systems whose gain coefficient varies in space.

*amostafazadeh@ku.edu.tr
A simple optical toy model that supports spectral singularities is an infinite slab gain medium of thickness $L$ that is aligned along the $x-y$ plane [10]. See Fig. 1 .

It is easy to check that the following is an exact solution of Maxwell's equations

$\vec{E}(z, t)=E e^{-i \omega t} \psi(z) \hat{e}_{x}, \quad \vec{B}(z, t)=-i \omega^{-1} E e^{-i \omega t} \psi^{\prime}(z) \hat{e}_{y}$,

where $E$ is a constant, $\hat{e}_{x}$ and $\hat{e}_{y}$ are the unit vectors pointing along the positive $x$ and $y$ axes, $\psi$ is a continuously differentiable solution of the Schrödinger equation,

$$
-\psi^{\prime \prime}(z)+v(z) \psi(z)=k^{2} \psi(z),
$$

$v$ is the potential defined by

$$
v(z):=\left\{\begin{array}{lll}
k^{2}\left[1-n(z)^{2}\right] & \text { for } & |z| \leqslant \frac{L}{2}, \\
0 & \text { for } & |z|>\frac{L}{2},
\end{array}\right.
$$

$k:=\omega / c$ is the wave number, and $n(z)$ is the complex refractive index of the gain medium.

If the gain medium is obtained by doping a host medium of refraction index $n_{0}$ and is modeled as a two-level atomic system with lower- and upper-level population densities $N_{l}$ and $N_{u}$, resonance frequency $\omega_{0}$, and damping coefficient $\gamma$, we have

$$
n^{2}=n_{0}^{2}-\frac{\hat{\omega}_{p}^{2}}{\hat{\omega}^{2}-1+i \hat{\gamma} \hat{\omega}},
$$

where $\hat{\omega}:=\omega / \omega_{0}, \hat{\gamma}:=\gamma / \omega_{0}, \omega_{p}^{2}:=\left(N_{l}-N_{u}\right) e^{2} /\left(m_{e} \varepsilon_{0}\right), e$ is electron's charge, and $m_{e}$ is its mass. It is not difficult to show that

$$
\hat{\omega}_{p}^{2}=2 \hat{\gamma} \kappa_{0} \sqrt{n_{0}^{2}+\kappa_{0}^{2}}, \quad \kappa_{0}:=-\frac{\lambda_{0} g_{0}}{4 \pi},
$$

where $\lambda_{0}:=2 \pi c / \omega_{0}$ is the resonance wavelength, and $g_{0}$ is the effective gain coefficient ${ }^{1}$ at the resonance frequency [10]. For all known gain media, $\kappa_{0} \ll n_{0}$. Therefore,

$$
\hat{\omega}_{p}^{2} \approx 2 \hat{\gamma} n_{0} \kappa_{0}=-\frac{\hat{\gamma} n_{0} \lambda_{0} g_{0}}{2 \pi} .
$$

\footnotetext{
${ }^{1}$ By effective gain coefficient we mean the gain coefficient minus the loss coefficient.
} 


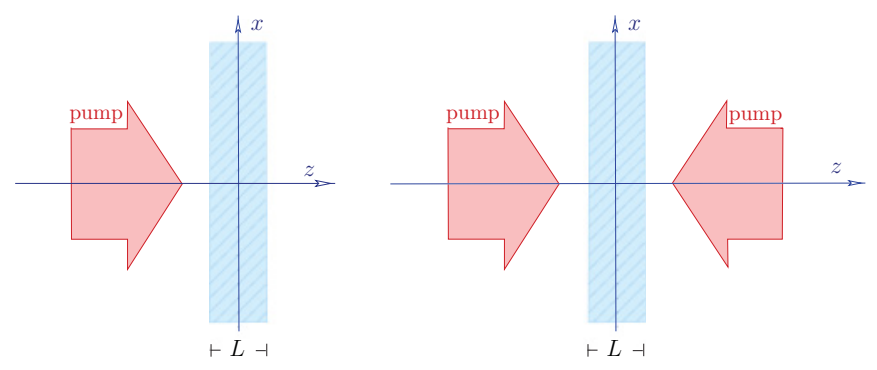

FIG. 1. (Color online) Cross section of an infinite planar slab gain medium [hatched (blue) region] in the $x-z$ plane. Arrows represent pumping beams. Left- and right-hand plots represent single and double-pumping, respectively.

In general the gain coefficient $g_{0}$ is a function of $z$ inside the gain medium. For example, if we produce the gain by pumping the medium from the left-hand side, the intensity of the pumping beam decays exponentially as it penetrates the medium, and we have

$$
g_{0}(z)=\left(g_{\star}+\alpha_{0}\right) e^{-\frac{L}{2 \ell}} e^{-\frac{z}{\ell}}-\alpha_{0} \text { for }|z|<\frac{L}{2},
$$

where $g_{\star}:=g_{0}(-L / 2)$ is the value of $g_{0}$ where the pumping beam enters the gain medium, and $\alpha_{0}$ is the absorption (attenuation or loss) coefficient at the resonance frequency. Note that the maximum gain coefficient $g_{\star}$ is attained upon population inversion. Hence $g_{\star} \leqslant \alpha_{0}$ [11]. If we pump the gain medium from both sides (double-pumping), we find

$$
g_{0}(z)=\left[\frac{g_{\star}+\alpha_{0}}{\cosh \left(\frac{L}{2 \ell}\right)}\right] \cosh \left(\frac{z}{\ell}\right)-\alpha_{0} \quad \text { for } \quad|z|<\frac{L}{2} .
$$

It is useful to formulate the problem in terms of the dimensionless coordinate variable:

$$
\mathrm{x}:=\frac{z}{L}+\frac{1}{2} .
$$

Then the Schrödinger equation, (1), takes the form

$$
-\Psi^{\prime \prime}(\mathrm{x})+\mathrm{V}(\mathrm{x}) \Psi(\mathrm{x})=\mathfrak{K}^{2} \Psi(\mathrm{x}),
$$

where $\Psi(\mathrm{x}):=\psi\left(L \mathrm{x}-\frac{L}{2}\right)$,

$$
\begin{aligned}
& \mathrm{V}(\mathrm{x}):=\left\{\begin{array}{lc}
\alpha+\beta \mathrm{G}_{0}(\mathrm{x}) & \text { for } 0 \leqslant \mathrm{x} \leqslant 1, \\
0 & \text { otherwise, }
\end{array}\right. \\
& \alpha:=\mathfrak{K}^{2}\left(1-n_{0}^{2}\right), \quad \beta:=\frac{n_{0} \hat{\gamma} \hat{\omega} \mathfrak{K}}{1-\hat{\omega}^{2}-i \hat{\gamma} \hat{\omega}}, \\
& \mathfrak{K}:=L k=\frac{2 \pi L \hat{\omega}}{\lambda_{0}}, \quad \mathrm{G}_{0}(\mathrm{x}):=L g_{0}\left(L \mathrm{x}-\frac{L}{2}\right) .
\end{aligned}
$$

We therefore consider the problem of finding the spectral singularities of the potentials of the form

$$
\mathrm{V}(\mathrm{x}):=\left\{\begin{array}{lc}
\mathfrak{z}_{1}+\mathfrak{z}_{2} f(\mathrm{x}) & \text { for } 0 \leqslant \mathrm{x} \leqslant 1, \\
0 & \text { otherwise, }
\end{array}\right.
$$

where $f:[0,1] \rightarrow \mathbb{R}$ is a piecewise twice-differentiable realvalued function, and $\mathfrak{z}_{1}$ and $\mathfrak{z}_{2}$ are complex coupling constants that can depend on $\mathfrak{K}$.

\section{TRANSFER MATRIX AND SPECTRAL SINGULARITIES}

The general solution of (9) has the form

$$
\Psi(\mathrm{x})=\left\{\begin{array}{lcc}
A_{-} e^{i \mathfrak{K} \mathrm{X}}+B_{-} e^{-i \mathfrak{K} \mathrm{X}} & \text { for } & \mathrm{x}<0, \\
A_{0} \Phi_{1}(\mathrm{x} ; \mathfrak{K})+B_{0} \Phi_{2}(\mathrm{x} ; \mathfrak{K}) & \text { for } & 0 \leqslant \mathrm{x} \leqslant 1, \\
A_{+} e^{i \mathfrak{K} \mathrm{X}}+B_{+} e^{-i \mathfrak{K} \mathrm{X}} & \text { for } & \mathrm{x}>1
\end{array}\right.
$$

where $A_{0}, B_{0}, A_{ \pm}, B_{ \pm}$are complex coefficients, and $\Phi_{1}(\cdot ; \mathfrak{K})$ and $\Phi_{2}(\cdot ; \mathfrak{K})$ are a pair of linearly independent solutions of (9) in the interval $[0,1]$. Imposing the condition that $\Psi$ and $\Psi^{\prime}$ are continuous at $\mathrm{x}=0$ and $\mathrm{x}=1$, we can relate $A_{+}$and $B_{+}$to $A_{-}$and $B_{-}$and determine the transfer matrix $\mathbf{M}$ of the system that satisfies $\vec{C}_{+}=\mathbf{M} \vec{C}_{-}$with $\vec{C}_{ \pm}:=\left(\begin{array}{c}A_{ \pm} \\ B_{ \pm}\end{array}\right)$.

As discussed in [4] and [7], the spectral singularities are the real values of $\mathfrak{K}$ for which the $M_{22}$ entry of the transfer matrix vanishes. The latter has the form

$$
M_{22}=\frac{e^{i \mathfrak{K}} F(\mathfrak{K})}{2 W},
$$

where $\quad W:=\Phi_{1}(\mathrm{x} ; \mathfrak{K}) \Phi_{2}^{\prime}(\mathrm{x} ; \mathfrak{K})-\Phi_{1}^{\prime}(\mathrm{x} ; \mathfrak{K}) \Phi_{2}(\mathrm{x} ; \mathfrak{K})$ is the Wronskian of $\Phi_{1}(\cdot ; \mathfrak{K})$ and $\Phi_{2}(\cdot ; \mathfrak{K})$,

$$
F(\mathfrak{K}):=i \mathfrak{K}\left[\Gamma_{1}^{-}(1 ; \mathfrak{K}) \Gamma_{2}^{+}(0 ; \mathfrak{K})-\Gamma_{2}^{-}(1 ; \mathfrak{K}) \Gamma_{1}^{+}(0 ; \mathfrak{K})\right],
$$

and

$$
\Gamma_{j}^{ \pm}(\mathrm{x} ; \mathfrak{K}):=\Phi_{j}(\mathrm{x} ; \mathfrak{K}) \pm(i \mathfrak{K})^{-1} \Phi_{j}^{\prime}(\mathrm{x} ; \mathfrak{K}), \quad j=1,2 .
$$

Next we fix the choice of the solutions $\Phi_{1}(\cdot ; \mathfrak{K})$ and $\Phi_{2}(\cdot ; \mathfrak{K})$ by demanding that they fulfill the initial conditions: ${ }^{2}$

$$
\begin{gathered}
\Phi_{1}(0, \mathfrak{K})=1, \quad \Phi_{1}^{\prime}(0, \mathfrak{K})=-i \mathfrak{K}, \\
\Phi_{2}(0, \mathfrak{K})=1, \quad \Phi_{2}^{\prime}(0, \mathfrak{K})=0 .
\end{gathered}
$$

In view of (17) and (18), we have $\Gamma_{1}^{+}(0 ; \mathfrak{K})=0$ and $\Gamma_{2}^{ \pm}(0 ; \mathfrak{K})=$ 1 . These, together with (15) and (16), imply that the spectral singularities of the potential, (13), are the real zeros of the function

$$
F(\mathfrak{K})=i \mathfrak{K} \Phi_{1}(1 ; \mathfrak{K})-\Phi_{1}^{\prime}(1 ; \mathfrak{K}) .
$$

Equating the right-hand side of this equation to 0 and solving for $\mathfrak{K}$ give

$$
\mathfrak{K}=-i G(1, \mathfrak{K}),
$$

where

$$
G(\mathrm{x}, \mathfrak{K}):=\frac{\Phi_{1}^{\prime}(\mathrm{x} ; \mathfrak{K})}{\Phi_{1}(\mathrm{x} ; \mathfrak{K})}=\frac{\partial}{\partial \mathrm{x}} \ln \left[\Phi_{1}(\mathrm{x} ; \mathfrak{K})\right] .
$$

Because we require $\mathfrak{K}$ to be real, (21) is equivalent to

$$
\begin{aligned}
\operatorname{Re}[G(1, \mathfrak{K})] & =0, \\
\operatorname{Im}[G(1, \mathfrak{K})] & =\mathfrak{K} .
\end{aligned}
$$

These are the basic real equations that determine the spectral singularities.

\footnotetext{
${ }^{2}$ This is an acceptable choice, because it gives $W=i \mathfrak{K} \neq 0$.
} 


\section{SEMICLASSICAL SPECTRAL SINGULARITIES}

In order to apply the above procedure of determining spectral singularities of the potentials of the form (13), we need an explicit expression for the solution $\Phi_{1}$ of the Schrödinger equation, (9). It is well known that except for the few exactly solvable special cases, there is no exact and explicit method of constructing such a solution. In this section we employ the method of semiclassical (WKB) approximation to determine $\Phi_{1}$ and employ the method of Sec. II.

The semiclassical solutions of (9) are given by the wellknown expression

$$
\Psi_{ \pm}(\mathrm{x} ; \mathfrak{K}):=R(\mathrm{x} ; \mathfrak{K}) \exp \left[ \pm i \int_{0}^{\mathrm{X}} \sqrt{\mathfrak{K}^{2}-\mathrm{V}(\mathrm{x})} d \mathrm{x}\right]
$$

where

$$
R(\mathrm{x} ; \mathfrak{K}):=\left[\mathfrak{K}^{2}-\mathrm{V}(\mathrm{x})\right]^{-1 / 4} .
$$

These solutions are reliable provided that we can neglect $R^{\prime \prime}(\mathrm{x} ; \mathfrak{K}) / R(\mathrm{x} ; \mathfrak{K})$ for all $\mathrm{x} \in[0,1]$, i.e.,

$$
\left|\frac{4\left[\mathfrak{K}^{2}-\mathrm{V}(\mathrm{x})\right] \mathrm{V}^{\prime \prime}(\mathrm{x})+5 \mathrm{~V}^{\prime}(\mathrm{x})^{2}}{16\left[\mathfrak{K}^{2}-\mathrm{V}(\mathrm{x})\right]^{3}}\right| \ll 1 .
$$

Here and in what follows we assume that $\mathrm{V}$ is a piecewise twice-differentiable function and (27) is imposed on the intervals in which $\mathrm{V}$ is twice-differentiable.

Because $\Phi_{1}(\cdot ; \mathfrak{K})$ is a solution of $(9)$, it must be a linear combination of $\Psi_{ \pm}(\cdot ; \mathfrak{K})$; there are complex numbers $A$ and $B$ such that

$$
\Phi_{1}(\mathrm{x} ; \mathfrak{K})=A \Psi_{+}(\mathrm{x} ; \mathfrak{K})+B \Psi_{-}(\mathrm{x} ; \mathfrak{K}) .
$$

We can determine the coefficients $A$ and $B$ by imposing the initial conditions (18). This gives

$$
\begin{aligned}
& A=\frac{1}{2 R_{0}}\left[1-\mathfrak{K} R_{0}^{2}+\frac{i}{4} R_{0}^{6} \mathrm{~V}^{\prime}(0)\right], \\
& B=\frac{1}{2 R_{0}}\left[1+\mathfrak{K} R_{0}^{2}-\frac{i}{4} R_{0}^{6} \mathrm{~V}^{\prime}(0)\right],
\end{aligned}
$$

where $R_{\mathrm{X}}:=R(\mathrm{x} ; \mathfrak{K})=\left[\mathfrak{K}^{2}-\mathrm{V}(\mathrm{x})\right]^{-1 / 4}$.

Next, we substitute (28) in (20), equate the resulting expression to 0 , and use (29) and (30) to derive the following relation for the spectral singularities.

$$
\begin{aligned}
\exp \left\{2 i \int_{0}^{1} \sqrt{\mathfrak{K}^{2}-\mathrm{V}(\mathrm{x})} d \mathrm{x}\right\} \\
=\frac{\left[1+\mathfrak{K} R_{0}^{2}-\frac{i}{4} \mathrm{~V}^{\prime}(0) R_{0}^{6}\right]\left[1+\mathfrak{K} R_{1}^{2}+\frac{i}{4} \mathrm{~V}^{\prime}(1) R_{1}^{6}\right]}{\left[1-\mathfrak{K} R_{0}^{2}+\frac{i}{4} \mathrm{~V}^{\prime}(0) R_{0}^{6}\right]\left[1-\mathfrak{K} R_{1}^{2}-\frac{i}{4} \mathrm{~V}^{\prime}(1) R_{1}^{6}\right]} .
\end{aligned}
$$

For the cases where $\mathrm{V}(0)=\mathrm{V}(1)$ and $\mathrm{V}^{\prime}(0)=-\mathrm{V}^{\prime}(1)$, Eq. (31) reduces to

$$
\exp \left\{2 i \int_{0}^{1} \sqrt{\mathfrak{K}^{2}-\mathrm{V}(\mathrm{x})} d \mathrm{x}\right\}=\left[\frac{1+\mathfrak{K} R_{0}^{2}-\frac{i}{4} \mathrm{~V}^{\prime}(0) R_{0}^{6}}{1-\mathfrak{K} R_{0}^{2}+\frac{i}{4} \mathrm{~V}^{\prime}(0) R_{0}^{6}}\right]^{2} .
$$

A simple example is the complex barrier potential $V(\mathrm{x})$, which has a constant value $\mathfrak{z}:=\mathfrak{K}^{2}\left(1-n^{2}\right)$ throughout the interval
$[0,1]$. For this potential, the semiclassical approximation is exact, and (32) takes the simple form

$$
e^{2 i \mathfrak{K} n}=\left(\frac{n+1}{n-1}\right)^{2} .
$$

This coincides with the exact result given in [10].

In the remainder of this article we explore the application of our general results for the potentials of the form (13). First, we introduce the following pair of variables:

$$
\mathfrak{r}:=\sqrt{1-\frac{\mathfrak{z}_{1}}{\mathfrak{K}^{2}}}, \quad \mathfrak{s}:=\frac{\mathfrak{z}_{2}}{\mathfrak{K}^{2}-\mathfrak{z}_{1}} .
$$

Using (13) and (34), we can express (31) as

$$
\exp \left\{2 i \mathfrak{r} \mathfrak{K} \int_{0}^{1} \sqrt{1-\mathfrak{s} f(\mathrm{x})} d x\right\}=\mathscr{E}(\mathfrak{K}, \mathfrak{r}, \mathfrak{s}),
$$

where

$$
\begin{aligned}
& \mathscr{E}(\mathfrak{K}, \mathfrak{r}, \mathfrak{s}):= {\left[\frac{1+\mathfrak{r} p_{0}(\mathfrak{s})-\mathfrak{K}^{-1} q_{0}(\mathfrak{s})}{1-\mathfrak{r} p_{0}(\mathfrak{s})-\mathfrak{K}^{-1} q_{0}(\mathfrak{s})}\right] } \\
& \times\left[\frac{1+\mathfrak{r} p_{1}(\mathfrak{s})+\mathfrak{K}^{-1} q_{1}(\mathfrak{s})}{1-\mathfrak{r} p_{1}(\mathfrak{s})+\mathfrak{K}^{-1} q_{1}(\mathfrak{s})}\right], \\
& p_{\mathrm{X}}(\mathfrak{s}):=\sqrt{1-\mathfrak{s} f(\mathrm{X})}, \quad q_{\mathrm{X}}(\mathfrak{s}):=\frac{i \mathfrak{s} f^{\prime}(\mathrm{X})}{4[1-\mathfrak{s} f(\mathrm{X})]} .
\end{aligned}
$$

Next, we take the logarithm of both sides of (35). In view of the multivaluedness of "ln," we can write (35) in the following equivalent form:

$$
\mathfrak{K}=\frac{2 \pi m+\arg [\mathscr{E}(\mathfrak{K}, \mathfrak{r}, \mathfrak{s})]-i \ln |\mathscr{E}(\mathfrak{K}, \mathfrak{r}, \mathfrak{s})|}{2 \mathfrak{r} \int_{0}^{1} \sqrt{1-\mathfrak{s} f(\mathrm{x})} d \mathrm{x}},
$$

where $m=0, \pm 1, \pm 2, \ldots$, and " $\arg [z] "$ stands for the principal argument of $z$. The integer $m$, which, in this way, enters into the calculation of spectral singularities, serves as a mode number. This provides a general explanation for the emergence of a mode number in the study of the spectral singularity-related resonance effect. ${ }^{3}$

Next, we recall that $\mathfrak{K}$ takes real values. Therefore the righthand side of (38) must be real. This allows us to write (38) as the following pair of real equations:

$$
\begin{gathered}
\{2 \pi m+\arg [\mathscr{E}(\mathfrak{K}, \mathfrak{r}, \mathfrak{s})]\} \rho(\mathfrak{r}, \mathfrak{s}) \\
+\ln |\mathscr{E}(\mathfrak{K}, \mathfrak{r}, \mathfrak{s})| \sigma(\mathfrak{r}, \mathfrak{s})=\mathfrak{K}, \\
\{2 \pi m+\arg [\mathscr{E}(\mathfrak{K}, \mathfrak{r}, \mathfrak{s})]\} \sigma(\mathfrak{r}, \mathfrak{s}) \\
-\ln |\mathscr{E}(\mathfrak{K}, \mathfrak{r}, \mathfrak{s})| \rho(\mathfrak{r}, \mathfrak{s})=0,
\end{gathered}
$$

where

$$
\begin{aligned}
\rho(\mathfrak{r}, \mathfrak{s}) & :=\operatorname{Re}\left[\left(2 \mathfrak{r} \int_{0}^{1} \sqrt{1-\mathfrak{s} f(\mathrm{x})} d \mathrm{x}\right)^{-1}\right], \\
\sigma(\mathfrak{r}, \mathfrak{s}) & :=\operatorname{Im}\left[\left(2 \mathfrak{r} \int_{0}^{1} \sqrt{1-\mathfrak{s} f(\mathrm{x})} d \mathrm{x}\right)^{-1}\right] .
\end{aligned}
$$

\footnotetext{
${ }^{3}$ In previous studies of the subject the emergence of such a mode number could only be linked to the properties of the specific functions entering the calculations $[4,8]$.
} 
In view of (40), we can express (39) as

$$
\mathfrak{K}=\left[\sigma(\mathfrak{r}, \mathfrak{s})+\frac{\rho(\mathfrak{r}, \mathfrak{s})^{2}}{\sigma(\mathfrak{r}, \mathfrak{s})}\right] \ln |\mathscr{E}(\mathfrak{K}, \mathfrak{r}, \mathfrak{s})| .
$$

As we see below, for the typical optical applications, $|\mathfrak{s}| \ll$ $1 \ll|\mathfrak{K}|$ and $|\mathfrak{r}| \approx n_{0}$. This observation has three important consequences. First, it implies that $m$ takes rather large positive values. Second, it confirms the validity of the semiclassical approximation (27). Third, it suggests that we can perform a reliable perturbative calculation of spectral singularities by choosing $\mathfrak{s}$ and $\mathfrak{K}^{-1}$ as perturbation parameters. ${ }^{4}$

\section{DOUBLE-PUMPING OF AN INFINITE SLAB GAIN MEDIUM}

As pointed out in Sec. I, double-pumping of an infinite slab gain medium corresponds to a complex potential of the form (13) with

$$
\begin{gathered}
\mathfrak{z}_{1}=\mathfrak{K}^{2}\left[n_{0}^{2}\left(\hat{g}_{\star} \mathfrak{t}-1\right)+1\right], \\
\mathfrak{z}_{2}=n_{0}^{2} \mathfrak{K}^{2}\left(\hat{g}_{\star}+1\right) \mathfrak{t}, \\
f(\mathrm{x})=\frac{\cosh \left[v\left(\mathrm{x}-\frac{1}{2}\right)\right]}{\cosh \left(\frac{v}{2}\right)}-1,
\end{gathered}
$$

where we have used (7) and (10)-(12) and introduced

$$
\hat{g}_{\star}:=\frac{g_{\star}}{\alpha_{0}} \leqslant 1, \quad \mathfrak{t}:=\frac{\hat{\gamma} \lambda_{0} \alpha_{0}}{2 \pi n_{0}\left(1-\omega^{2}-i \hat{\gamma} \hat{\omega}\right)}, \quad v:=\frac{L}{\ell} .
$$

Clearly, $v=0$ corresponds to the case where the gain coefficient is uniform throughout the medium. Using (44)-(46) in (37), (36), and (34), we find

$$
\begin{gathered}
\mathscr{E}(\mathfrak{K}, \mathfrak{r}, \mathfrak{s})=\left[\frac{\mathfrak{r}+1+\frac{i \nu \tanh \left(\frac{v}{2}\right) \mathfrak{s}}{4 \mathfrak{K}}}{\mathfrak{r}-1-\frac{i \nu \tanh \left(\frac{v}{2}\right) \mathfrak{s}}{4 \mathfrak{K}}}\right]^{2}, \\
\mathfrak{r}=n_{0} \sqrt{1-\hat{g}_{\star} \mathfrak{t},}, \mathfrak{s}=\frac{\left(1+\hat{g}_{\star}\right) \mathfrak{t}}{1-\hat{g}_{\star} \mathfrak{t}} .
\end{gathered}
$$

Now consider a typical semiconductor gain medium [11] with

$$
\begin{gathered}
n_{0}=3.4, \quad \lambda_{0}=1500 \mathrm{~nm}, \quad \hat{\gamma}=0.02, \quad \alpha_{0}=200 \mathrm{~cm}^{-1}, \\
L \approx 300 \mu \mathrm{m}, \quad g_{\star} \approx 50 \mathrm{~cm}^{-1}, \quad v \approx 0.1 .
\end{gathered}
$$

Then we find that at resonance frequency, $\mathfrak{K}=\mathfrak{K}_{0}:=$ $2 \pi L / \lambda_{0}>1250, \quad|\mathfrak{s}| \approx|\mathfrak{t}|<1.8 \times 10^{-3}, \quad$ and $\left|\mathfrak{r}-n_{0}\right|<$ $6.0 \times 10^{-4}$. These numerical bounds suggest that $\mathfrak{K}^{-1}, \mathfrak{s}$, and $\mathfrak{t}$ are suitable perturbation parameters for an accurate perturbative calculation of spectral singularities. ${ }^{5}$ The same is also true in the vicinity of the resonance frequency, $\hat{\omega} \approx 1$,

\footnotetext{
${ }^{4}$ In a first-order perturbative calculation, in which we ignore the quadratic and higher order terms in $\mathfrak{K}^{-1}$ and $\mathfrak{s}, \mathfrak{K}^{-1} q_{0}$, and $\mathfrak{K}^{-1} q_{1}$ drop from the right-hand side of (36), $\mathscr{E}$ becomes $\mathfrak{K}$-independent, and Eqs. (39) and (40) decouple.

${ }^{5}$ Note that $\mathfrak{K}=\hat{\omega} \mathfrak{K}_{0}$.
}

and for other typical gain media where $\lambda_{0} / L \ll 1$ and $\lambda_{0} g_{\star} \leqslant$ $\lambda_{0} \alpha_{0} \ll 1$. Furthermore, we can check that for this sample the left-hand side of (27) is of the order of $10^{-8}$. Therefore, semiclassical approximation provides an extremely accurate solution of the problem.

Having related the parameters of the problem to the relevant physical data, we wish to investigate the consequences of imposing (40) and (43), which ensure the emergence of a spectral singularity. Because of the complicated nature of these equations, this can only be done numerically. It turns out, however, that an approximate perturbative treatment can play a vital role in elucidating the physical content of these equations.

\section{A. Perturbation theory and a universal bound on lasing}

In this section we perform a perturbative treatment of spectral singularities that involves using $\mathfrak{K}^{-1}$ and $\mathfrak{t}$ as perturbation parameters. This is a particularly appropriate choice, because as we stated above, $\left|\mathfrak{K}^{-1}\right| \approx|\mathfrak{t}| \approx 10^{-3}$.

First, we examine the results of the first-order perturbation theory where we ignore quadratic and higher order terms in $\mathfrak{K}^{-1}$ and $\mathfrak{t}$. In particular, because $\mathfrak{s}$ is proportional to $\mathfrak{t}$, we ignore terms involving $\mathfrak{s} / \mathfrak{K}$ in (48). This gives

$$
\begin{aligned}
\mathscr{E}(\mathfrak{K}, \mathfrak{r}, \mathfrak{s}) \approx & \left(\frac{n_{0}+1}{n_{0}-1}\right)^{2}\left[1+\frac{2 n_{0} \hat{g}_{\star} \operatorname{Re}(\mathfrak{t})}{n_{0}^{2}-1}\right] \\
& \times \exp \left(\frac{2 i n_{0} \hat{g}_{\star} \operatorname{Im}(\mathfrak{t})}{n_{0}^{2}-1}\right) .
\end{aligned}
$$

Note that in this case $\mathscr{E}(\mathfrak{K}, \mathfrak{r}, \mathfrak{s})$ does not depend on $\mathfrak{K}$. As a result, (40) and (43) decouple; (40) determines the location of the spectral singularities, and (43) gives the corresponding value of $\mathfrak{K}$.

Next, we use (41), (42), (46), and (49) to compute $\rho$ and $\sigma$. Again ignoring quadratic and higher order terms in $\mathfrak{t}$, we find

$$
\rho \approx \frac{1+\eta \operatorname{Re}(\mathfrak{t})}{2 n_{0}}, \quad \sigma \approx \frac{\eta \operatorname{Im}(\mathfrak{t})}{2 n_{0}},
$$

where

$$
\eta:=\frac{\left(1+\hat{g}_{\star}\right) \tanh \left(\frac{v}{2}\right)}{v}-\frac{1}{2} .
$$

It proves useful to examine the case $\hat{\omega}=1$ separately. In this case,

$$
\begin{gathered}
\mathfrak{t}=\frac{i \lambda_{0} \alpha_{0}}{2 \pi n_{0}}, \quad \rho \approx \frac{1}{2 n_{0}}, \quad \sigma \approx \frac{\lambda_{0} \alpha_{0} \eta}{4 \pi n_{0}^{2}}, \\
\mathscr{E}(\mathfrak{K}, \mathfrak{r}, \mathfrak{s}) \approx\left(\frac{n_{0}+1}{n_{0}-1}\right)^{2} \exp \left[\frac{i \lambda_{0} g_{\star}}{\pi\left(n_{0}^{2}-1\right)}\right] .
\end{gathered}
$$

Now we are in a position to impose (40) and (43). These, respectively, give

$$
\begin{gathered}
\eta \approx\left(\frac{2 n_{0}}{\lambda_{0} \alpha_{0} m}\right) \ln \left(\frac{n_{0}+1}{n_{0}-1}\right), \\
\mathfrak{K}_{0} \approx \frac{\pi m}{n_{0}} .
\end{gathered}
$$


We can use the latter relation to obtain the mode number for the spectral singularity at resonance wavelength. The result is

$$
m \approx \frac{2 n_{0} L}{\lambda_{0}}=1360,
$$

where we have used $\mathfrak{K}_{0}=2 \pi L / \lambda_{0} \approx 1256.637$ and the numerical values (50) and (51). ${ }^{6}$

Note that because $\mathfrak{K}_{0}>0,(58)$ implies that $\eta>0$. In light of (54), this is equivalent to $\left(\hat{g}_{\star}+1\right)^{-1} \lesssim \frac{2}{v} \tanh \left(\frac{v}{2}\right)$. Moreover, we know that $\hat{g}_{\star} \leqslant 1$ and $\frac{2}{v} \tanh \left(\frac{v}{2}\right) \leqslant 1$. Combining these inequalities, we find

$$
\frac{1}{2} \leqslant \frac{1}{1+\hat{g}_{\star}} \lesssim \frac{2 \tanh \left(\frac{v}{2}\right)}{v} \leqslant 1 .
$$

In particular, $4 \tanh \left(\frac{v}{2}\right)-v \geqslant 0$. This puts an upper bound on the value of the damping constant $v$, namely, $v \lesssim 3.83$. Equivalently, the total damping factor satisfies

$$
1-e^{-v} \lesssim 0.978 \text {. }
$$

It is remarkable that this bound is independent of other physical parameters of the system. Note also that because producing a spectral singularity at the resonance frequency requires a smaller gain than a spectral singularity with a different frequency, this bound applies more generally for $\hat{\omega} \approx 1$. More importantly, in view of the fact that spectral singularities saturate the laser threshold condition [10], (61) is actually a universal bound on the possibility of lasing in any doubly pumped gain medium.

Next, we insert (54) and (59) in (57) and solve for $g_{\star}=$ $\hat{g}_{\star} \alpha_{0}$. This yields

$$
g_{\star} \approx \frac{v}{\tanh \left(\frac{\nu}{2}\right)}\left[\frac{1}{L} \ln \left(\frac{n_{0}+1}{n_{0}-1}\right)+\frac{\alpha_{0}}{2}\right]-\alpha_{0} .
$$

Figure 2 shows a plot of the right-hand side of (62), which turns out to be an increasing function of $v$ for $v \geqslant 0 .^{7}$

In view of the fact that $g_{\star}$ cannot exceed $\alpha_{0}$, (62) implies $v \lesssim v_{\max }$ where $v_{\max }$ satisfies

$$
2 \tanh \left(\frac{v_{\max }}{2}\right)-\left[\frac{1}{\alpha_{0} L} \ln \left(\frac{n_{0}+1}{n_{0}-1}\right)+\frac{1}{2}\right] \nu_{\max }=0 .
$$

Solving this equation numerically we find the following improved bounds:

$$
\begin{aligned}
v & \lesssim v_{\max } \approx 3.01714112 \\
1-e^{-v} & \lesssim 1-e^{-v_{\max }} \approx 0.9510590651 .
\end{aligned}
$$

The latter relation means that in order to realize a spectral singularity at the resonance frequency, the intensity of each of the pumping beams should not drop to less than $e^{-v_{\max }} \approx 4.9 \%$ of its value in vacuum as it traverses the gain medium. This corresponds to a $95.1 \%$ loss, which is much larger than in typical lasing media. ${ }^{8}$

\footnotetext{
${ }^{6}$ In principle, there is no reason for this calculation to yield an integer value for $m$. Indeed it gives $m \approx 1359.9999999999998$ !

${ }^{7}$ This is actually to be expected, because it means that for larger values of the decay constant $v$, we need larger gain coefficients to maintain the same spectral singularity.

${ }^{8}$ Typically the loss is less than $10 \%$.
}

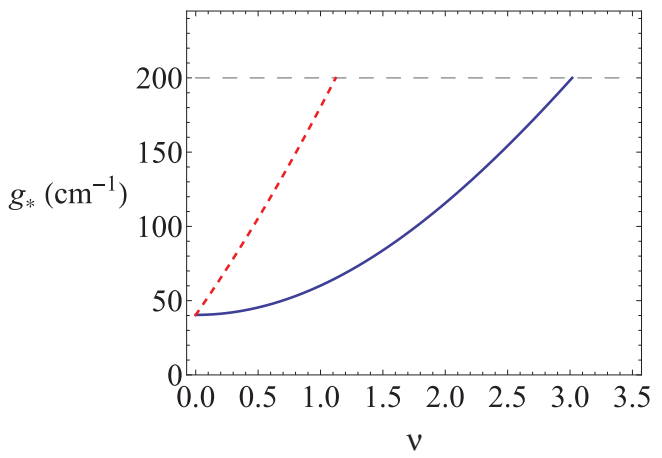

FIG. 2. (Color online) A plot of the gain coefficient $g_{\star}$ necessary for creating a spectral singularity at the resonance wavelength as a function of the damping constant $v$ for the doubly pumped sample considered in Sec. IV [solid (blue) curve] and singly pumped sample considered in Sec. V [dashed (red) curve]. The horizontal dashed (gray) line marks the upper bound on $g_{\star}$.

Another implication of (62) is that in the absence of damping $(v=0)$, the minimum gain coefficient necessary for creating a spectral singularity (at the resonance frequency) is given by

$$
g_{\star}=\frac{2}{L} \ln \left(\frac{n_{0}+1}{n_{0}-1}\right) \approx 40.40905 \mathrm{~cm}^{-1} .
$$

This is in complete agreement with the results of [10].

Next, we recall from [10] that the presence of a spectral singularity is extremely sensitive to the values of the parameters of the system. This suggests that the above first-order perturbative results may not provide a sufficiently accurate description of the spectral singularities; we need to carry out at least a second-order perturbative calculation in which we also account for the quadratic terms in $\mathfrak{t}$ and $\mathfrak{K}^{-1}$. Here we summarize the resulting expressions:

$$
\begin{gathered}
|\mathscr{E}(\mathfrak{K}, \mathfrak{r}, \mathfrak{s})| \approx\left(\frac{n_{0}+1}{n_{0}-1}\right)^{2}\left[1+C_{1} \operatorname{Re}(\mathfrak{t})+C_{2} \operatorname{Re}(\mathfrak{t})^{2}\right. \\
\left.+C_{3} \operatorname{Im}(\mathfrak{t})^{2}+\frac{C_{4} v \tanh \left(\frac{v}{2}\right) \operatorname{Im}(\mathfrak{t})}{\mathfrak{K}}\right], \\
\arg [\mathscr{E}(\mathfrak{K}, \mathfrak{r}, \mathfrak{s})] \approx C_{1} \operatorname{Im}(\mathfrak{t})-2 C_{3} \operatorname{Re}(\mathfrak{t}) \operatorname{Im}(\mathfrak{t}) \\
-\frac{C_{4} v \tanh \left(\frac{v}{2}\right) \operatorname{Re}(\mathfrak{t})}{\mathfrak{K}}, \\
\rho \approx \frac{1}{2 n_{0}}\left[1+\eta \operatorname{Re}(\mathfrak{t})+3 \xi \operatorname{Re}(\mathfrak{t})^{2}-3 \xi \operatorname{Im}(\mathfrak{t})^{2}\right], \\
\sigma \approx \frac{1}{2 n_{0}}[\eta \operatorname{Im}(\mathfrak{t})+6 \xi \operatorname{Re}(\mathfrak{t}) \operatorname{Im}(\mathfrak{t})],
\end{gathered}
$$

where

$$
\begin{gathered}
C_{1}:=\frac{2 n_{o} \hat{g}_{\star}}{n_{0}^{2}-1}, \quad C_{2}:=\frac{\left(3 n_{0}^{2}+4 n_{0}-1\right) n_{0} \hat{g}_{\star}^{2}}{2\left(n_{0}^{2}-1\right)^{2}}, \\
C_{3}:=-\frac{n_{0}\left(3 n_{0}^{2}-1\right) \hat{g}_{\star}^{2}}{2\left(n_{0}^{2}-1\right)^{2}}, \quad C_{4}:=-\frac{n_{0}\left(1+\hat{g}_{\star}\right)}{n_{0}^{2}-1}, \\
\xi:=\frac{1}{8}\left\{1+\frac{\left(1+\hat{g}_{\star}\right)\left[1+\hat{g}_{\star}+\left(\hat{g}_{\star}-3\right)\left(\frac{\sinh v}{v}\right)\right]}{\cosh v+1}\right\} .
\end{gathered}
$$


Inserting (66)-(69) in (40), noting that $2 \pi m$ is of the same order of magnitude as $\mathfrak{K}$, and keeping the three lowest order terms in the pertubative expansion, we obtain

$$
\begin{gathered}
\pi m \eta \operatorname{Im}(\mathfrak{t})-\ln \left(\frac{n_{0}+1}{n_{0}-1}\right)+6 \pi m \xi \operatorname{Re}(\mathfrak{t}) \operatorname{Im}(\mathfrak{t}) \\
-\left[\frac{n_{0} \hat{g}_{\star}}{n_{0}^{2}-1}+\eta \ln \left(\frac{n_{0}+1}{n_{0}-1}\right)\right] \operatorname{Re}(\mathfrak{t}) \approx 0 .
\end{gathered}
$$

Doing the same for (39) gives

$$
\begin{aligned}
\mathfrak{K} \approx & \frac{1}{n_{0}}\left\{\pi m\left[1+\eta \operatorname{Re}(\mathfrak{t})+3 \xi \operatorname{Re}(\mathfrak{t})^{2}-3 \xi \operatorname{Im}(\mathfrak{t})^{2}\right]\right. \\
& \left.+\left[\frac{n_{0} \hat{g}_{\star}}{n_{0}^{2}-1}+\eta \ln \left(\frac{n_{0}+1}{n_{0}-1}\right)\right] \operatorname{Im}(\mathfrak{t})\right\} .
\end{aligned}
$$

If we examine the spectral singularity at the resonance frequency, we find that (70) reduces to (57), while (71) yields the following improvement of (58):

$$
\mathfrak{K}_{0} \approx \frac{\pi m}{n_{0}}+\frac{\lambda_{0} \alpha_{0}}{2 \pi n_{0}^{2}}\left[\left(\eta-\frac{3 \xi}{\eta}\right) \ln \left(\frac{n_{0}+1}{n_{0}-1}\right)+\frac{n_{0} \hat{g}_{\star}}{n_{0}^{2}-1}\right] .
$$

\section{B. Numerical results}

Here we report the results of a numerical treatment of Eqs. (39) and (40). This involves fixing the values of $n_{0}, \lambda_{0}, \hat{\gamma}$, and $\alpha_{0}$ as given by (50), setting $L=300 \mathrm{~nm}$, and determining $\lambda$ and $g_{\star}$ for various choices of the decay constant $\nu$. Here is a summary of our findings.

(1) The numerical results agree with the results of secondorder perturbative calculations to at least nine significant figures.

(2) It turns out that increasing $v$ starting from its minimum value $v=0$ (unform gain coefficient) has an extremely small effect on the wavelength of the spectral singularities. We find spectral singularities with almost the same wavelengths but, as expected, with larger values of the gain coefficient $g_{\star}$. Table I lists the values of $m, \lambda$, and $g_{\star}$ for different $\nu$.

(3) For $v \leqslant v_{1} \approx 0.22519975$ there are precisely 55 spectral singularities, corresponding to mode numbers $m=$ $1333,1334, \ldots, 1387$. The values of the wavelength $\lambda$ and gain coefficient $g_{\star}$ for these spectral singularities are depicted in Fig. 3. As expected, the spectral singularity with the least amount of gain is the one generated at the resonance wavelength.

(4) Figure 3 provides a clear demonstration of how one can use spectral singularities to generate a tunable laser [10]. By controlling the intensity of the pumping beam we can adjust $g_{\star}$ to produce lasing at any of the 55 wavelengths shown in Fig. 2. These turn out to be almost equally spaced in the range 1471.2-1529.9 nm, with an average spacing of $1.07 \mathrm{~nm}$.

(5) As one increases $v$ beyond $v_{1} \approx 0.22519975$ the number of spectral singularities starts to decrease. This is because in this case in order to create the spectral singularity with mode number $m=1335$ or 1387 , the system requires a larger gain coefficient $g_{\star}$ than $\alpha_{0}=200 \mathrm{~cm}^{-1}$. This is not possible, for $\alpha_{0}$ is the largest value that $g_{\star}$ can take. As

\begin{tabular}{|c|c|c|c|c|c|}
\hline \multicolumn{3}{|c|}{$m=1335$} & \multicolumn{3}{|c|}{$m=1360$} \\
\hline$v$ & $\lambda(\mathrm{nm})$ & $g_{\star}\left(\mathrm{cm}^{-1}\right)$ & $v$ & $\lambda(\mathrm{nm})$ & $g_{\star} \mathrm{cm}^{-1}$ \\
\hline 0.0 & 1527.6859891 & 175.59110 & 0.0 & 1499.9999833 & 40.40905 \\
\hline 0.1 & 1527.6859888 & 175.90413 & 0.1 & 1499.9999831 & 40.60936 \\
\hline 0.2 & 1527.6859881 & 176.84258 & 0.2 & 1499.9999826 & 41.20988 \\
\hline 0.3 & 1527.6859868 & 178.40459 & 0.3 & 1499.9999819 & 42.20942 \\
\hline 0.5 & 1527.6859827 & 183.38565 & 0.5 & 1499.9999794 & 45.39683 \\
\hline \multicolumn{3}{|c|}{$m=1350$} & \multicolumn{3}{|c|}{$m=1380$} \\
\hline$v$ & $\lambda(\mathrm{nm})$ & $g_{\star}\left(\mathrm{cm}^{-1}\right)$ & $v$ & $\lambda(\mathrm{nm})$ & $g_{\star}\left(\mathrm{cm}^{-1}\right)$ \\
\hline 0.0 & 1510.9539613 & 61.80307 & 0.0 & 1478.5584532 & 124.17655 \\
\hline 0.1 & 1510.9539612 & 62.02123 & 0.1 & 1478.5584530 & 124.44660 \\
\hline 0.2 & 1510.9539607 & 62.67527 & 0.2 & 1478.5584524 & 125.25620 \\
\hline 0.3 & 1510.9539598 & 63.76387 & 0.3 & 1478.5584514 & 126.60373 \\
\hline 0.5 & 1510.9539570 & 67.23530 & 0.5 & 1478.5584482 & 130.90084 \\
\hline
\end{tabular}

TABLE I. Values of wavelength $\lambda$ and gain coefficient $g_{\star}$ for spectral singularities of the doubly pumped sample considered in Sec. IV with $m=1335,1350,1360$, and 1380 for different damping coefficients $v$.

one increases $v$ further the number of spectral singularities keeps dropping. For $v>v_{\max } \approx 3.01714279$ the last spectral singularity (namely, the one at the resonance wavelength) cannot be maintained either. This observation is in good agreement with the bound, $v \lesssim 3.01714112$, that we found using the first-order perturbative calculations.

\section{SINGLE PUMPING OF AN INFINITE SLAB GAIN MEDIUM}

Consider pumping of the semiconductor slab gain medium studied in the preceding section from the left-hand side (see Fig. 1). In this case, the gain coefficient $g_{0}$ and the parameters $\mathfrak{z}_{1}, \mathfrak{z}_{2}, \mathfrak{r}$, and $\mathfrak{s}$ are given by (6), (44), (45), and (49), respectively, while the function $f$, which describes the space dependence of the gain coefficient, takes the form

$$
f(\mathrm{x})=e^{-v \mathrm{X}}-1
$$

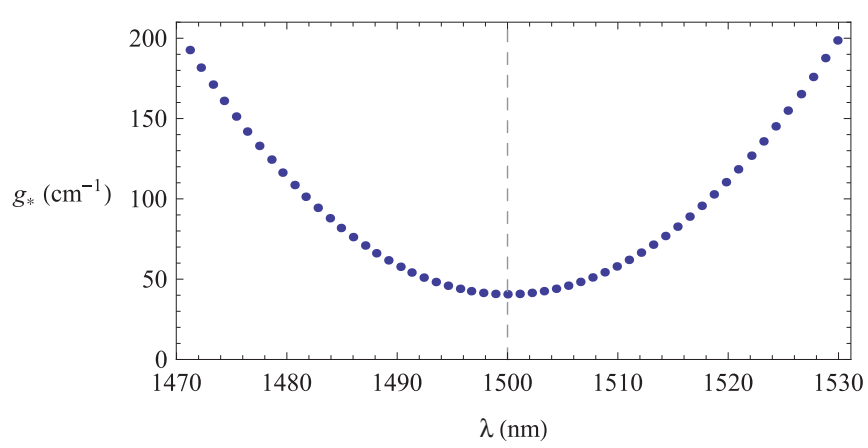

FIG. 3. (Color online) Location of the spectral singularities of the doubly pumped sample considered in Sec. IV in the $g_{\star}-\lambda$ plane for $v \leqslant 0.22519975$. The 55 data points correspond to decreasing values of the mode number $m$ from 1387 to 1333 . The dashed line marks the resonance wavelength $\lambda=\lambda_{0}=1500 \mathrm{~nm}$. 
This in turn implies

$$
\begin{aligned}
\mathscr{E}(\mathfrak{K}, \mathfrak{r}, \mathfrak{s}) & =\left(\frac{\mathfrak{r}+1+\frac{i \nu \mathfrak{s}}{4 \mathfrak{K}}}{\mathfrak{r}-1-\frac{i \nu \mathfrak{s}}{4 \mathfrak{K}}}\right) \\
& \times\left(\frac{\mathfrak{r} \sqrt{1+\left(1-e^{-v}\right) \mathfrak{s}}+1-\frac{i \nu \mathfrak{s}}{4 \mathfrak{K}\left[1+\left(1-e^{-v}\right) \mathfrak{s}\right]}}{\mathfrak{r} \sqrt{1+\left(1-e^{-v}\right) \mathfrak{s}}-1+\frac{i v \mathfrak{s}}{4 \mathfrak{K}\left[1+\left(1-e^{-\nu}\right) \mathfrak{s}\right]}}\right),
\end{aligned}
$$

where we have used (36) and (37).

Performing a first-order perturbative calculation of $\rho$ and $\sigma$ yields (53), with $\eta$ given by

$$
\eta:=\frac{\left(1-e^{-v}\right)\left(1+\hat{g}_{\star}\right)}{2 v}-\frac{1}{2} .
$$

In particular, at resonance frequency $\hat{\omega}=1$, we find that (55) still holds but (56) is slightly modified:

$$
\mathscr{E}(\mathfrak{K}, \mathfrak{r}, \mathfrak{s}) \approx\left(\frac{n_{0}+1}{n_{0}-1}\right)^{2} \exp \left[\frac{i \lambda_{0} g_{\star} \zeta}{\pi\left(n_{0}^{2}-1\right)}\right],
$$

where $\zeta:=\frac{1}{2}\left[1+e^{-v}-\hat{g}_{\star}^{-1}\left(1-e^{-v}\right)\right]$. Substituting (55) and (76) in (40) and (43), we recover (57) and (58). Again, we can use these equations and (75) to obtain the gain coefficient $g_{\star}$ as a function of $v$. The result is

$$
g_{\star} \approx \frac{2 v}{1-e^{-v}}\left[\frac{1}{L} \ln \left(\frac{n_{0}+1}{n_{0}-1}\right)+\frac{\alpha_{0}}{2}\right]-\alpha_{0} .
$$

The right-hand side of this relation is also an increasing function of $v$. This, together with the fact that $g_{\star}$ cannot exceed $\alpha_{0}$, puts an upper bound on the allowed values of $\nu$. Requiring the right-hand side of (77) not to be larger than $\alpha_{0}$ gives

$$
\frac{1-e^{-v}}{v} \gtrsim \frac{1}{\alpha_{0} L} \ln \left(\frac{n_{0}+1}{n_{0}-1}\right)+\frac{1}{2}>\frac{1}{2} .
$$

If we enforce the weaker condition, $\left(1-e^{-v}\right) / v>1 / 2$, we find the following numerical bounds on the decay constant and decay factor:

$$
v \lesssim 1.6, \quad 1-e^{-v} \lesssim 0.80 .
$$

Because these are independent of the parameters of the system, they apply generally for any singly pumped gain medium. If we enforce the stronger condition, namely, the first inequality in (78), we find

$$
\begin{gathered}
v \lesssim v_{\max } \approx 1.12208974, \\
1-e^{-v} \lesssim 1-e^{-v_{\max }} \approx 0.67440134 .
\end{gathered}
$$

These are in extremely good agreement with the (exact) numerical treatment of spectral singularities, which gives

$$
\begin{gathered}
v \lesssim v_{\max } \approx 1.12209007, \\
1-e^{-\nu} \lesssim 1-e^{-v_{\max }} \approx 0.67440144 .
\end{gathered}
$$

A comparison of these relations with (64) shows that the bounds for the doubly pumped sample are much weaker than those for the singly pumped sample, as is to be expected.

Another outcome of our numerical investigation is that the wavelength of the spectral singularities are very close to those obtained for the doubly pumped sample of Sec. IV. However, to create them one needs higher gain coefficients, particularly as $v$ increases (This is clearly displayed in Fig. 2 for the spectral singularity at the resonance frequency.) Again, the maximum number of spectral singularities that one can create is 55, and they correspond to mode numbers 13331387. All of these can be created provided that $v \leqslant v_{1} \approx$ $8.435993 \times 10^{-3}$. This corresponds to a damping of less than $1-e^{-v_{1}} \approx 8.40050985 \times 10^{-3}<0.85 \%$.

\section{SUMMARY AND CONCLUSION}

In realistic optical models that display spectral singularities the gain coefficient is a function of space. This motivates the study of the mathematical problem of finding spectral singularities for potentials that vanish outside a closed interval. In this article we have identified spectral singularities with real zeros of a particular complex-valued (so-called Jost) function, derived a semiclassical expression for this function, and used it to locate the spectral singularities of a typical semiconductor gain medium that is subject to either single or double-pumping. In both cases, we performed highly reliable pertutbative calculations and compared them with the exact numerical results.

The approach pursued here is particularly effective, because it turns out that, for the typical optical realizations of spectral singularities, the semiclassical approximation provides an excellent description. An important outcome of this approach is the fact that the inclusion of the effects of the exponential decay of the intensity of the pumping beams as they pass through the gain medium does not alter the wavelengths of the spectral singularities significantly. This seems to be an indication of the stability of spectral singularities. The mathematical origin of this behavior may be traced to the fact that spectral singularities are zeros of complex analytic functions.

An interesting but expected feature of the inclusion of the decay of the pumping beams is that the gain coefficient $g_{\star}$ associated with a given spectral singularity is an increasing function of the decay parameter $v$. This, combined with the observation that $g_{\star}$ is bounded from above by the absorption coefficient $\alpha_{0}$, implies the existence of an upper bound $v_{\max }$ on $v$ beyond which no spectral singularity can be produced. This is actually a bound on any kind of lasing, because spectral singularities saturate the lasing threshold condition. Using first-order perturbation theory we obtained a numerical value for $v_{\max }$ that is independent of the physical parameters of the system. Therefore, $v_{\max }$ is a universal upper bound on $v$ beyond which no lasing occurs.

As one steadily increases $v$ starting from $v=0$, one encounters a number of critical values $v_{j}$ with $j=1,2, \ldots$. at which the number of allowed spectral singularities drops by one. This phenomenon may have interesting ramifications for the application of spectral singularities in producing tunable lasers. For the gain medium we considered, there is a maximum of 55 spectral singularities. Double- and single pumping of this sample give 0.22519975 and 0.00843993 , respectively, for the first critical value of $v$, namely, $v_{1}$. Clearly, $v_{\max }=v_{55}$. 


\section{ACKNOWLEDGMENTS}

I wish to thank Aref Mostafazadeh and Ali Serpengüzel for illuminating discussions. This work was supported by the Scientific and Technological Research Council of Turkey (TÜBITAK) in the framework of Project No. 110T611 and by the Turkish Academy of Sciences (TÜBA).
[1] By diagonalizability of a non-Hermitian Schrödinger operator $H:=-\frac{d^{2}}{d x^{2}}+v(x)$ with spectrum $[0, \infty)$, we mean the existence of pairs of functions $\psi_{k}$ and $\phi_{k}$ satisfying $H \psi_{k}=k^{2} \psi_{k}, H^{\dagger} \phi_{k}=k^{2} \phi_{k}, \int_{\infty}^{\infty} \psi_{k}(x)^{*} \phi_{k^{\prime}}(x) d x=\delta(K-$ $\left.K^{\prime}\right)$, and $\int_{-\infty}^{\infty} \psi_{k}(x)^{*} \phi_{k}\left(x^{\prime}\right) d k=\delta\left(x-x^{\prime}\right),[12]$.

[2] If the spectrum includes a spectral singularity, one can regularize the last relation in [1] to reinstate the completeness of the eigenfunctions of $H$. For a recent discussion see A. A. Andrianov, F. Cannata, and A. V. Sokolov, J. Math. Phys. 51, 052104 (2010).

[3] M. A. Naimark, Trudy Moscov. Mat. Obsc. 3, 181 (1954) [Am. Math. Soc. Transl. 16(2), 103 (1960)]; R. R. D. Kemp, Can. J. Math. 10, 447 (1958); J. Schwartz, Commun. Pure Appl. Math. 13, 609 (1960); G. Sh. Guseinov, Pramana. J. Phys. 73, 587 (2009).

[4] A. Mostafazadeh, Phys. Rev. Lett. 102, 220402 (2009).
[5] A. Mostafazadeh, J. Math. Phys. 43, 2814 (2002); 43, 3944 (2002); C. M. Bender, D. C. Brody, and H. F. Jones, Phys. Rev. Lett. 89, 270401 (2002); A. Mostafazadeh and A. Batal, J. Phys. A 37, 11645 (2004); for a comprehensive review see [12].

[6] A. Mostafazadeh, J. Phys. A 39, 13495 (2006).

[7] A. Mostafazadeh and H. Mehri-Dehnavi, J. Phys. A 42, 125303 (2009).

[8] A. Mostafazadeh, Phys. Rev. A 80, 032711 (2009).

[9] Z. Ahmed, J. Phys. A 42, 472005 (2009); S. Longhi, Phys. Rev. B 80, 165125 (2009); Phys. Rev. A 81, 022102 (2010).

[10] A. Mostafazadeh, Phys. Rev. A 83, 045801 (2011).

[11] W. T. Silfvast, Laser Fundamentals (Cambridge University Press, Cambridge, 1996).

[12] A. Mostafazadeh, Int. J. Geom. Methods Mod. Phys. 7, 1191 (2011), e-print arXiv:0810.5643. 